\section{EL SISTEMA EDUCATIVO DE LOS PRIMEROS JESUITAS}

\author{
Josep María Margenat Peralta \\ Universidad Loyola Andalucía \\ margenat@uloyola
}

\section{PRIMITIVE JESUIT'S EDUCATIONAL SYSTEM}

Cómo citar este artículo/Citation: Margenat Peralta, J. M. (2016). El sistema educativo de los primeros jesuitas. Arbor, 192 (782): a356. doi: http://dx.doi.org/10.3989/arbor.2016.782n6001
Copyright: (C) 2016 CSIC. Este es un artículo de acceso abierto distribuido bajo los términos de la licencia Creative Commons Attribution (CC BY) España 3.0.

Recibido: 04 noviembre 2014. Aceptado: 25 agosto 2015.

RESUMEN: Entre los primeros documentos de las universidades jesuitas de mediados del siglo XVI (entre 1551 y 1586) -y la versión más consolidada y difundida de la Ratio de Acquaviva de 1599- se observan importantes diferencias, señaladas ya en su día por Gabriel Codina y por Miquel Batllori. Es así que este artículo, a partir del estado de la cuestión y de investigaciones anteriores del autor, pretende poner de relieve el núcleo original de la "invención" renacentista de los primeros colegios y universidades jesuitas. La metodología propuesta por Luce Giard que considera la complejidad reticular de un colegio y universidad jesuita como lugares de producción y de difusión el saber, y no solo ni principalmente como centros de transmisión (educativa), permite una aproximación ante los logros culturales de una modernidad iniciada en el siglo XVI, inspirada en un permanente diálogo entre los saberes y las creencias. El artículo tiende a presentar este modelo como uno de los logros destacables de la primera modernidad europea configurada por los centros educativos jesuitas.

PALABRAS CLAVE: Acomodación discernida; colegios; fronteras; Jeroni Nadal; matriz ignaciana; ratio studiorum; universidades.
ABSTRACT: Among the first documents from the Jesuit universities from the middle of the 16th century (between 1551 and 1586) - and the most consolidated and disseminated version in the Ratio of Acquaviva from 1599 - there are important differences, already identified in their day by Gabriel Codina and by Miquel Batllori. This article, based on previous research by the author and also the state of play, aims to highlight the original nucleus of the Renaissance "invention" of the first Jesuit schools and universities. The methodology proposed by Luce Giard considers the lattice-like complexity of a Jesuit school and University as places of production and dissemination of knowledge and not only, or mainly, as centers of (educational) transmission. It offers an approximation to the cultural achievements of a modernity began in the 16th century, inspired by a permanent and previous dialogue (here the adjectives are essential) between knowledge and beliefs. The article presents this model as one of the most important achievements of the first European modernity established by the Jesuit schools.

KEYWORDS: Discerned accommodation; schools; borders; Jeroni Nadal; Ignatian matrix; ratio studiorum; universities. 


\section{INTRODUCCIÓN: ORIGINALIDAD DE LOS COLEGIOS JESUITAS EN UN TIEMPO DE CRISIS CULTURALES}

La gran creación de los primeros jesuitas fueron los colegios y universidades, ya que no siempre es posible diferenciar entre unos y otras. Sabemos ya que la originalidad de estas instituciones no descansó ni en que los jesuitas fuesen los primeros en el tiempo, ni en sus prácticas pedagógicas, sino en la trabazón interna de experiencias anteriores que dieron lugar a una poderosa síntesis, todavía hoy admirada y vigorosa.

El historiador catalán Batllori, continuando en la senda abierta por el profesor vasco de la Universidad Gregoriana, Pedro de Leturia, se preguntaba en los años 80 si Ignacio era un hombre medieval o renacentista (Batllori, 1997). Los colegios son una buena muestra del alcance de esta cuestión, pues, aun tratándose de una institución medieval en su origen, durante el Renacimiento sufrieron una gran transformación. De la misma forma que san Ignacio estaba informado por ambas condiciones, la medieval y la renacentista, también los colegios jesuitas reflejan esta convivencia, no exenta de tensiones, bien representadas por las diferencias entre los colegios parisinos en que vivió y estudió Ignacio y alguno de los primeros compañeros: Saint-Jacques, Montaigu y Sainte-Barbe. Los jesuitas no inventaron de la nada un modelo, sino que sintetizaron lo mejor de tradiciones precedentes: lo nuevo fue la síntesis y la espiritualidad, ésta sí "moderna" y que animaba la obra.

La primera mitad del siglo XVI fue un tiempo de cambios profundos, de crisis culturales duraderas. Al "otoño de la edad media" siguió la primavera de los studia humanitatis. En la época en que Ignacio comenzó sus estudios universitarios en Barcelona y los continuó en París y Venecia, se produce un "cambio epocal" en las coordenadas geográficas, pues a la nueva configuración del mundo mediterráneo, que pasó de ser un espacio abierto a espacio compartimentado, se superpuso la primera gran mundialización de relaciones viajeras, mercantiles y técnico-científicas que se había iniciado a finales del siglo XV con la expansión marítima primero de Portugal y, más tarde, de Castilla. Ciere del espacio mediterráneo y primacía del espacio euro-atlántico son el marco en que emerge una nueva relación entre el saber, la cultura y la ciencia.

Dos grandes tensiones se configuraron en este cambio epocal: entre las diferentes creaciones de matrices culturales, la espiritualidad de la naciente Compañía de Jesús y la misión que de ella brota es una muestra (Senent de Frutos, 2014), por una parte, de la ordenación en relación con el territorio de las que se va perfilando a mediados del siglo XVI. En un momento de expansión colonial y de explotación mercantilista de los recursos, de una primera mundialización o configuración del sistema-mundo (Immanuel Wallerstein), la Compañía representa el polo de "adaptación discernida" frente a una ordenación del territorio como fuente de recursos apropiables y como espacio que hay que ordenar socialmente desde la disciplina. Esta forma de ordenación del espacio, estatal en la configuración geopolítica del mundo económico y de la acción política, tuvo su correlato en la ordenación eclesiástica (O'Malley, 1996).

En primer lugar, observamos en la primera Compañía una aproximación integral a la realidad, no escindida ni reducida. La matriz ignaciana espiritual, los Ejercicios, hacen posible, suponen y aún exigen, un modo de proceder generador de relaciones de integración social (Senent de Frutos, 2014), pues se trata de una espiritualidad radical, que va a la raíz (la relación de Dios con su criatura) y que por tanto puede ser matriz de un disenso en la fidelidad, o de una fidelidad creativa que sólo crece por medio de una ortodoxia heterodoxa (Mongini, 2012).

En segundo lugar, la propuesta de dos tipos ideales, a la manera weberiana, de dos modelos que nos sirven para comprender la reforma católica: el modelo ignaciano y el modelo tridentino. El segundo se configuró a partir de la parroquia como lugar que organiza un territorio; incluso las tierras que no están divididas en diócesis y en parroquias son tierras que van a depender de una congregación que tenía sus vicariatos apostólicos para "ordenar" los territorios "extraterritoriales", si podemos hablar así. Si la tradición tridentina obró de afuera hacia dentro, la ignaciana lo hizo de dentro afuera. La primera, la tridentina, regulaba legalmente el ministerio eclesial y la vida de los fieles con el objetivo de la disciplina externa; la segunda tradición se basaba en la experiencia religiosa misma y consideraba su profundización y su autenticidad como "la piedra de toque" de todo lo demás. Pedro y Pablo, como es tantas veces señalado. Los colegios y universidades jesuitas siguieron este segundo modelo de acomodación discernida en un sistema permanente de fronteras móviles (hacia fuera y, lo que es mucho más importante, hacia dentro y hacia abajo). Disenso y movilidad discernida son, pues, las claves de esta acomodación ignaciana.

En la transición entre el mundo romano y la ruptura de la "ekuméme" mediterránea para dar nacimiento a la Europa carolingia se configuraron en el centro de Europa tres formas "romanes" (románicas) con 
las que los europeos aprendimos a convivir y a comportarnos unos con otros: parroquia, casa y cofradía. Estas formas fueron naciendo a partir del siglo $\mathrm{V}$ y se formaron hasta la ruptura de la unidad carolingia en el siglo IX, con el desplazamiento hacia el centro y oriente europeo de la gravedad europea. En aquellos tres siglos y medio se fraguaron las tres formas básicas en que los europeos nos hemos venido relacionando y configurando: la parroquia, la iglesia propia y la confraternidad. La parroquia es el espacio de lo próximo, lo territorial entendido como configurador de la relación. La parroquia se ocupa de registrar el nacimiento y la defunción, de inscribir en la línea de duración la propia biografía. La parroquia agrupa a los que viven cerca de una casa (del griego "pará" y "oikía”). El diezmo acercaba a la casa ("oikía") como expresión del pacto parroquial, y a partir de él, en Europa se generó una cultura del pacto que creaba obligación, solidaridad, vinculación social. Esta primera forma nació en la transición del siglo $\mathrm{V}$ como protección ante la incertidumbre generada por lo deshabitado, lo desconocido y lo hundido. Más tarde esta forma dio lugar al pacto social, "estatal", el pacto de los próximos ante la inseguridad. La forma parroquial es la forma estatal. La segunda forma nacida también entre el siglo IV y el VI es la alternativa -en la que aún hoy nos movemos-: la búsqueda de la seguridad y de las oportunidades de beneficio en la protección privada, la que surge del que puede más sobre el que puede menos. El que puede más concede un "beneficio" para su servicio y el de los que se acogen a él: nacen los negocios "propios", las que defienden la lógica del interés particular en competencia con otros particulares, es la lógica de la casa, del patrimonio, de la herencia. El mercado regulador de los intercambios es su forma moderna, que hoy ha alcanzado indebidas e inquietantes dimensiones. La tercera forma nacida también en la transición de los siglos VI y VII es la que elige la pobreza comunitaria como respuesta de solidaridad ante la crisis y la inseguridad. El nacimiento del monacato y, más tarde, de las "confrèries", conlleva una confianza puesta en la reciprocidad del don de unos a otros. Esa forma se basa en la pobreza compartida que genera una fraternidad distinta de la "fratría" derivada de la vinculación a la casa y distinta a su vez de la "solidarietas" de la proximidad parroquial. Este espacio de cooperación es el que ha tardado más en desarrollarse. El modelo inspirado en la matriz ignaciana parte de una espiritualidad radical que funciona como matriz común del disenso y de la fidelidad. A las tres "formas" europeas medievales, la espiritualidad ignaciana contribuye con una apertura flexible a lo nuevo, ("novum"), como algo distinto, no algo a someter, sino a reconocer en su diferencia, entendiendo su lógica y sus pretensiones (Ejercicios espirituales, $\mathrm{n}$ 응 22): es la forma frontera ("limes") en la que funciona una verdadera fraternidad con los otros en cuanto "socios", no sólo en cuanto "próximos". Los colegios y universidades son un lugar de creación y ordenación de esa acomodación discernida en el saber y en el creer a partir del diálogo poroso, permeable en la instalación/desinstalación.

La diferencia entre los saberes de ambos tiempos -medieval y renacentista moderno- estribaba en el paso de la cultura como comentario y contemplación, en la que la dimensión transcendente de lo real era un dato incuestionado, que integraba la visión humanista con la propia auctoritas de quien se refería el texto, al saber como una aproximación empírica a partir de la observación que ha de ser verificada en lo real. El texto en sí desplazaba al comentador del mismo, y éste a su vez era superado y quizá suplantado por la observación exterior; la relación entre lo real y el que aprende era ya directa, positiva, y no estaba limitada por la escuela, no era una mera repetición -es este caso propiamente llamada escolástica.

Hasta entonces la enseñanza se basaba en la lectio y privilegiaba la transmisión oral. Ahora en lugar del texto era toda la realidad la que se podía leer, observar y descifrar. Al aprendizaje memorístico sucedía la lectura, la exégesis de los textos, así como la praelectio (método combinado de presentación, análisis y síntesis). Este desplazamiento había de llegar a su final con la llustración, y con esto a sus últimas consecuencias. Fue Nietzsche quien mejor formuló un siglo más tarde esta absolutización del horizonte humanoantropocéntrico, al hablar de la sombra que Dios -un "dios" ique estaba muerto!- dejaba tras su paso: tal como es la especie humana, durante milenios habrá cavernas en las que tal vez se mostrará su sombra.

Existe un horizonte humanista no antropocéntrico, sino teocéntrico o como otros denominan de una teonomía referenciada o de una autonomía teónoma. Ignacio nos propone un itinerario integral humanista que no procede a las reducciones limitantes de la comprensión de ciertas líneas directrices del pensamiento moderno. El pensador hodierno que ha formulado con más profundidad y alcance este humanismo ha sido, a nuestro juicio, Jacques Maritain, al acuñar al expresión "humanismo integral" para referirse a un verdadero humanismo que no es antropocéntrico, sino un humanismo referenciado (teónomo), pero secular y pluralista (Maritain, 1936/2000). 


\section{LA NOVEDAD DEL SISTEMA EDUCATIVO JESUITA}

¿Qué hizo san Ignacio con todo este proceso de cambios? Él, que no fue un brillante intelectual ni pretendió ser un humanista, aunque sus diez años de serios estudios en Barcelona y París le dieron una sólida formación, consiguió sin embargo, una nueva síntesis humanista teocéntrica. Ignacio destacó como el organizador que prevé un nuevo estatuto en los colegios, un nuevo estatus para la formación de los jóvenes en el humanismo letrado y abierto a la trascendencia, en piedad y en letras. Por ese motivo, los colegios jesuitas fueron especialmente próximos a las aspiraciones e intereses culturales de las nuevas élites sociales urbanas de Europa, de una Europa que empezaba a descomponer su unidad política y religiosa, y en la que emergían entre Augsburgo y Westfalia, ahora ya con un prevalerte contenido político, las viejas nationes medievales. Esta cercanía a las élites no les impidió hacerse todo con todos, especialmente los más pobres, en quienes veían privilegiadamente a Cristo (Margenat, 2013). El proceso de deculturación que siguió Ignacio le llevó a pensar un nuevo modelo de formación común a seglares y clérigos, lo que era coherente con la primigenia inspiración de los Ejercicios espirituales, su obra fundamental que marca una espiritualidad nueva para el miles christianus, para el bautizado, independientemente de su condición clerical o seglar.

En la actualidad, unos tres mil jesuitas trabajan en las casi doscientas cuarenta instituciones universitarias y de enseñanza superior. Casi desde sus comienzos, la Compañía de Jesús se vio inmersa en la misión universitaria. Ese casi tiene su importancia, ya que en los planes iniciales de Ignacio de Loyola y sus primeros compañeros, maestros en Artes por la de París, no entraron sin embargo las universidades. Ellos se reconocían a sí mismos como clérigos instruidos y libremente pobres, llamados a una vocación apostólica e itinerante, no a la enseñanza. Las universidades, estables corporaciones de estudiantes y maestros de plurales naciones, no eran su horizonte. El primer grupo estaba formado por hombres de frontera, que eran de "nationes variae" (cántabros -uno gamboíno y otro oñacino-, castellanos, saboyanos, además de un picardo, un portugués y un provenzal). El horizonte de inteligibilidad en que aquel grupo de universitarios inscribía su proyecto no era el de una estabilidad que proporcionara beneficios y prelaturas, sino la frontera. Ésta se vio modificada y alterada por otra frontera, esta vez cultural: el diálogo con los saberes de cada tiempo y de cada lugar. Entre las primeras fundaciones de residencias para jóvenes estudiantes jesuitas de París y Coímbra, todavía excluyendo que la propia Compañía dirigiese establecimientos escolares como tales, y la creación, a petición del Senado de la ciudad de Mesina, del "primum ac prototypum collegium" en 1548 , se produjo un cambio esencial en la propia constitución estructurante de la Compañía de Jesús. Este giro se reflejó inmediatamente en su máximo texto normativo y orientativo, las Constituciones.

Los primeros jesuitas querían que sus colegios fuesen espacios escolares autónomos, independientes de otras instituciones tutelares. Esta importancia atribuida a los colegios hizo que muy pronto se desarrollase una importante red de enseñanza secundaria y universitaria en gran parte de Europa central y occidental, así como en la América castellana y portuguesa y en las Indias orientales. Estos colegios pasaron de ser lugares de residencia, como hasta entonces eran concebidos, a ser lugares de cultura. En los primeros años (1540-1548) los jesuitas fundaron colegios como residencias para los jóvenes estudiantes de la orden en universidades como París, pronto trasladado a Lovaina, Padua, Coímbra, Alcalá de Henares (Castilla), Colonia y Valencia. Más tarde, con los colegios de Gandía (Valencia) y de Goa, los jesuitas comenzaron a enseñar en sus colegios a estudiantes seglares, junto a los jóvenes estudiantes jesuitas: en Goa, a cristianos, portugueses o no, de aquella ciudad india y en Gandía a población morisca. Poco después en Mesina (Sicilia) se fundó el primer colegio que perfeccionó el modelo de Goa y Gandía.

Según el historiador jesuita Ganss, al morir san Ignacio (1556) entre los 40 colegios existentes había al menos seis modelos: residencias sólo de estudiantes jesuitas que asistían a los cursos en la universidad, como las citadas; residencias como las anteriores en las que profesores jesuitas daban algunos cursos para los estudiantes jesuitas; un tercer modelo, similar al anterior aunque ya con oyentes externos, como el de Gandía desde 1546; colegios como el de Mesina, concebidos para estudiantes externos, seglares, aunque con la presencia de algunos jesuitas como alumnos; seminarios para la formación de futuros clérigos, como el Colegio Germánico de Roma (1552); y, por último, residencias exclusivamente para seglares sin aspiración a las órdenes sagradas, como el de Viena (1553). El que tendrá más continuidad y expansión, como es bien sabido, fue el modelo del colegio de Mesina.

"La Compañía de Jesús nació en un ambiente universitario" (Kolvenbach, 2008, 142). La educación universitaria fue la matriz que estructuró a la Compañía de Jesús, aunque no de forma directa, sino mediatamente. 
Ignacio de Loyola fue un hombre del que ha podido decirse que era, al mismo tiempo, medieval y renacentista (Batllori, 1997). En su vida pasó de las mediaciones cortas (peregrinar a Jerusalén y vivir a la apostólica) a las mediaciones largas (estudiar para "ayudar" a las personas). En Barcelona, a sus 33 años (febrero de 1524), "comunicó su inclinación de estudiar" con el maestro Jeroni Ardévol, que enseñaba latín en el Estudio General de la ciudad (este Studium generale precedió, como institución educativa superior, a la propiamente conocida como universidad de Barcelona, creada en 1533). Su formación universitaria propedéutica fue barcelonesa. En esa ciudad sabemos que no sólo aprendió latín, sino que frecuentó círculos lulianos y erasmistas, de lo que quedó una perceptible influencia. A la delicada formación de un cortesano castellano, recibida en Arévalo y Valladolid, unía la formación en letras humanas en un culto ambiente urbano. A los dos años catalanes siguieron la breve estancia complutense y los siete años de París, donde obtuvo el título de Maestro en Artes. Allí fue agrupando en torno a sí a unos pocos estudiantes, más jóvenes que él, germen de la primera "mínima Compañía de Jesús".

La primera experiencia de "compañía" fue la de unos universitarios. Se trató, sin embargo, de una experiencia utopista, germinada en una ciudad universitaria, exigente pero poco prometedora. Por eso se atreve a sentenciar Kolvenbach: “La vocación pedagógica y humanística de los primeros jesuitas parece accidental" (Kolvenbach, 2008). Ni Ignacio, ni tampoco el navarro Francisco Javier, el castellano Diego Laínez o el saboyano Pierre Favre, alimentaban el más mínimo proyecto de dedicarse a la educación humanística. "El entorno universitario parece haber tenido poco impacto en la concepción del apostolado de los primeros compañeros", llega a afirmar Kolvenbach (Kolvenbach, 2008, p. 35); y añade que Ignacio "se enfrentó frecuentemente a la cultura de su tiempo", y que la primera Compañía fue, en cierto sentido, "antiintelectual".

Esta determinación primera de los jesuitas lleva al historiador a la pregunta del porqué, en tan pocos años, de la opción por los colegios, que entonces incluían sin casi distinción a los centros universitarios, como sigue ocurriendo, aún hoy, en el vocabulario usado en los países anglosajones. Todavía en 1541 lgnacio excluía una dedicación docente para los jesuitas ("no studios ni lectiones en la Compañía"), pero ya en 1548 enviaba a Palermo un equipo de lo más selecto de la naciente Compañía, a cuyo frente ponía al mallorquín Jeroni Nadal, un universitario de París que se había incorporado más tarde al grupo. Los primeros jesuitas no podían prever el impacto que la aventura universitaria había de tener sobre la naciente Compañía, sobre las personas y sobre la estructura de la misma. Ésta había de vivir la misma evolución que la personal de Ignacio: de las mediaciones cortas ("predicar en pobreza") se iba a pasar a las mediaciones largas, en la complejidad y la densidad del mundo urbano, la naciente cultura científica y la economía mercantilista.

Este cambio implicaba el paso de aquellos "sacerdotes reformados", y reformadores sobre todo a través de la predicación y de un nuevo modo de vida y de reformar las costumbres. Sólo que, en aquel tiempo, cualquier proyecto de reforma exigía un retorno a la pobreza. Para aquellos "amigos en el Señor", "presbíteros de Cristo libremente pobres", maestros en artes, "pobres... pero instruidos", ésta era una tensión no sólo constituyente de su grupo o compañía, sino constitutiva de lo que había de ser hasta hoy la historia y el proyecto de la mínima Compañía. Ésta nació como una orden con un máximo de libertad, con una estricta pobreza y la coherente gratuidad de su trabajo, pero, en pocos años, una dedicación prioritaria a la enseñanza trastocó todo su sistema originario de pobreza, y aún todo el sistema institucional. Ignacio vio que, en ese momento, era más importante la dedicación a la enseñanza que a la predicación. La Compañía cambió radicalmente. Kolvenbach ha llegado a comparar este cambio con el giro radical que ha supuesto para la orden el compromiso por la justicia:

De la misma manera que la promoción de la justicia está cambiando la vida de la Compañía hoy día, la opción preferencial de fundar colegios y universidades afectó profundamente la Compañía recién nacida (Kolvenbach, 2008, p. 36).

Este cambio afectó a la naciente Compañía al menos en tres aspectos: la pobreza apostólica y la gratuidad de ministerios, el nuevo modo de gobierno centralizado e institucionalizado y, por último, el aminorado empuje misional. La creación de las universidades no era un fin en sí mismo, sino un medio, aunque, pues Ignacio sabía que "una universidad es una universidad", que tiene su finalidad propia, y no "mera oportunidad para la evangelización", nunca pretendió instrumentalizar el medio para otro fin (Kolvenbach, 2008, p. 37).

En pocos años, en los que la Compañía de Jesús cambió más que en los casi cuatrocientos años que van de la muerte de Ignacio en 1556 a la elección del venerado padre Arrupe como prepósito general en 1965, una decisión accidental, ni prevista, ni inicial- 
mente planeada, conllevó la transformación de una orden religiosa, creada para la frontera, en una red de instituciones muy estables, concebidas como "mediaciones largas" y estructuras de universalidad que integraban las "fronteras" como lugar/no lugar en que desarrollar un nuevo modo de proceder de acomodación discernida desde una matriz cultural nueva, en movimiento. Aunque la historia, vista desde la actualidad o de espaldas, nos lleva a veces a la afirmación de que la Compañía ha sido uno de los más potentes agentes culturales creadores de la educación secundaria y superior en la Europa moderna, la misma historia vista de cara, se nos presenta de otra forma. Una decisión accidental de la primera generación fue la que creó la verdadera estructura de la Compañía de Jesús tal como la conocemos. Es bien sabido que la primera concepción de Ignacio fue la de reunir y formar un grupo de hombres dedicados enteramente a discurrir por el mundo (que para ellos se configura como espacio sagrado sin fronteras, como un ager dominicus que ha de ser roturado: un ager que ha de convertirse en rus), para predicar a la apostólica y en pobreza, viviendo de las limosnas y no de rentas estables. No entraba en los planes de Ignacio la residencia estable en un lugar, a no ser por razones estratégicas o coyunturales. Sin embargo Laínez escribió en 1560:

la Compañía considera dos formas de ayudar a nuestro prójimo: uno en los colegios, por medio de la educación de la juventud en las letras, la enseñanza y la vida cristiana; otro, doquiera, asistiendo a todo tipo de personas (Monumenta historica Societatis lesu, MHSI en adelante).

La primera Fórmula de la naciente Compañía de Jesús (1540) consideraba toda la tierra como lugar propio de su misión, discurriendo de una parte a otra del mundo que, según solía decir Jeroni Nadal, es "nuestra casa". A esto añadía el jesuita mallorquín que las mejores casas de los jesuitas eran la "peregrinaciones” y las “misiones” (O’Malley, 1995, pp. 92-93, y las referencias a MHSI). Un tiempo después, el documento estatutario fundamental, las Constituciones, abrió tímidamente la posibilidad de que algunos residieran establemente en algunos sitios y, en pocos años, nos encontramos con una Compañía que dedicaba ingentes energías humanas y materiales a los colegios abiertos en Asia, Europa y América. La evolución siguió lenta, pero inexorablemente: pocos años antes de la supresión pontificia de 1773 , algo más de dos tercios de los 23000 jesuitas de todo el mundo eran educadores. En los 800 colegios de esta poderosa red mundial recibían educación en letras, enseñanza y vida cristiana unos 200000 alumnos. Unos dos siglos y medio más tarde el número de centros de educación se había doblado y el de alumnos se había multiplicado por ocho, para llegar a los actuales 1660000 . La Compañía seguía siendo una orden esencialmente docente. A este contingente de alumnos de primaria y secundaria, habría de sumarse al medio millón largo de estudiantes de educación superior, ya anotado.

Aunque el inicial grupo de París estaba constituido por hombres mayores, bien formados, pronto se unieron muchos jóvenes, que habían de prepararse en humanidades, filosofía, teología. Para ellos se abrieron colegios cercanos a universidades, como ya tenían otras órdenes, y se hizo una excepción en la severa regla de pobreza, permitiendo que las fundaciones de los colegios tuviesen rentas estables (Sauvé, Codina y Escalera, 2001). En pocos años se suceden los establecimientos: París (1540), al que siguieron el fundado por Juan III de Portugal en Coímbra (1542), los de Padua, Lovaina (1542), Colonia, Valencia (1544) y, unos años más tarde, Córdoba (1553) y Sevilla (1554).

En pocos años los colegios cambiaron mucho. En 1542 Javier escribe que en Goa los jesuitas han sido encargados de un colegio-seminario, en el que hay seglares y, desde 1545, hay jesuitas enseñando; también ese año, en los estados patrimoniales del duque de Gandía, se abre un colegio sólo para jóvenes que, muy probablemente, no habían de ser jesuitas, entre otras razones, porque, como la mayoría de la población de Gandía en ese momento, los jóvenes eran moriscos musulmanes; un año más tarde comienzan los alumnos externos, por lo que dice Nadal que el colegio de Gandía fue "el primero en el que los nuestros dieron clases abiertas al público"; en Coímbra, en 1547, y en Padua, dos años más tarde, los jesuitas comienzan a dar clases a jóvenes estudiantes jesuitas. Las dotaciones económicas para los colegios se muestran pronto insuficientes. La "revolución de los precios" (Hamilton) afectó también a las rentas patrimoniales estables de las fundaciones que, en poco tiempo, se revelaban patentemente escasas, debido a la inflación de los precios, causada por la abundancia del oro y la plata americanos, mientras las rentas fijas se devaluaban rápidamente.

El senado de Mesina pidió a la Compañía que fundase un colegio abierto, para lo que, en 1548, Ignacio envió un grupo de diez jesuitas, con los que nació el primer colegio jesuita para externos. En él, conocido como primum ac prototypum de los colegios jesuitas, tiene sus orígenes la Ratio studiorum institutionisque (el modo y orden de enseñanza y formación). El Colegio Romano, abierto en 1551, no es el primero crono- 
lógicamente, pero pronto se convirtió en el prototypum para toda la orden, tanto por su curriculum (su ratio studiorum) y los métodos allí establecidos, como por la filosofía educativa que lo inspiró.

En 1556, a la muerte de Ignacio, se ha impuesto la prioridad apostólica de los colegios y las universidades, tal como lo había formulado el secretario burgalés y judío de Ignacio, Juan de Polanco: "todo jesuita debe poner de su parte a la hora de contribuir a llevar el peso de los colegios". En 1555 Polanco había escrito que Ignacio deseaba que "los colegios se multipliquen más que las casas" y que no le importaba aceptar grandes cambios en el mismo interior de la Compañía para hacer sitio a ese compromiso educativo. En 1556, efectivamente, eran 46 los colegios abiertos (scholae publicae) y sólo dos las casas profesas, en las que la "Compañía profesa" vivía el primitivo ideal de la predicación apostólica en pobreza.

La capacidad de síntesis de la tercera generación de jesuitas permitió alumbrar el más importante proyecto educativo de la modernidad europea, la Ratio studiorum, tras los primeros balbuceos sicilianos. Ésta tuvo varias versiones, formuladas sucesivamente: la aún bastante más renacentista de 1586, en los primeros años de Aquaviva, es decir, más humanista y crítica, tras la que se llegó en 1599, a la formulación barroca, definitiva y consolidada por muchos siglos, plenamente adecuada a las posiciones del general jesuita Aquaviva y por ello mucho más contrarreformista. La Ratio, un curriculum único y básico, con el que se entrelaza un "modo de proceder", había de ser común a todos los colegios y universidades jesuitas, en muchos sitios hasta después de la segunda guerra mundial. Tres raíces, que reflejaban la propia formación de Ignacio y de los primeros jesuitas, alimentaban esta Ratio studiorum: la tradición formativa escolástica, humanismo flamenco y humanismo italiano. Por un lado, resultado de la experiencia común de todos ellos, ejercía un fuerte influjo el modus parisiensis, es decir la forma escolástica de enseñar y aprender usada en Sorbona; por otra parte, los trazos procedentes del modelo humanista introducido en algunos colegios de París, como el de Sainte-Barbe, que frecuentó Ignacio desde 1530, que seguía las orientaciones de los hermanos de la doctrina común, la corriente de la devotio moderna de los Países Bajos, que Ignacio ya conocía profundamente tras su prolongado contacto con el monasterio de Montserrat, foco de irradiación de la devotio en la Corona de Aragón; en la Ratio influyó, por último, el modus italicus, propio de la tradición humanista italiana, que ponía el acento en el estudio positivo de los padres de la Iglesia y la lectura de la Escritura.
La Compañía de Jesús concibió las Universidades desde su imagen moderna de la realidad. Ante un mundo en cambio, era y es necesario mantener una actitud de itinerancia, cambio adaptado, discernimiento continuo y vida a la apostólica. John W. O’Malley afirma que

el ministerio de los primeros jesuitas procedía de una experiencia de Dios completamente personal en su relación con cada criatura, de una acción de Dios "siempre mayor" de lo que se esperaba [y] precisamente porque la experiencia ignaciana se centra en la experiencia directa de Dios, los ministerios ignacianos están explorando constantemente nuevas fronteras (O'Malley, 1995, pp. 73-118 y O'Malley, 1996).

Por ello, frente al modelo tridentino de reforma eclesial y de disciplina social (Sozialdisciplinierung, según la expresión de G. Oestreich), es decir, procesos de crecimiento de las instituciones centralizadas y jerárquicas que transformaron el orden social y el espacio institucional, los jesuitas, presentaban el modelo de acomodación discernida y el voto de movilidad en las fronteras. Esto les llevó a crear desde muy al principio, aunque sin haberlo previsto, un ministerio nuevo y sin precedentes: los colegios, con el que abrieron una nueva era en la Iglesia, especialmente cuando, a finales del siglo XVI y en todo el XVII, fueron imitados por otras órdenes y congregaciones, especialmente algunas femeninas. Como he intentado esbozar, no sólo la Compañía concibió la acción apostólica a través de colegios y universidades, sino que también éstos dieron forma a la naciente Compañía de Jesús, lo que creó un conjunto de tensiones fecundas: los jesuitas habían de ser pobres, pero instruidos; vivían en la ciudad, tanto en el centro, allá donde se puede influir, como en las periferias urbanas o culturales; discurrían libremente pobres de una parte a otra, pero admitieron rentas para fundar colegios estables.

\section{HUMANISMO Y EDUCACIÓN EN LOS ESCRITOS EDU- CATIVOS DE JERONI NADAL}

San Ignacio siguió muy de cerca la elaboración de las Constituciones del primer colegio propiamente dicho de la Compañía de Jesús, en el que admitían estudiantes externos, pues había escrito que para Gandía se siguiese el ejemplo de lo realizado para Mesina:

"Siga el ejemplo del padre Nadal, quien, obtenida la facultad [de erigir el colegio] compuso las Constituciones del Colegio de Mesina y las envió a Roma para que las aprobaran" (Monumenta Historica Societatis lesu, a partir de ahora MHSI). 
L. Luckács, el editor de Monumenta paedagogica, en adelante Mon. Paed., en 1965, subrayaba por esta razón que Ignacio las consideraba modélicas, mientras se aprobaban unas comunes a todos los colegios ("el P. Nadal [...] ha ordenado algunas constituciones buenas", escribía Polanco por encargo de Ignacio a Araoz, 9-VII-1549, cfr. MHSI, Epistulae Mixtae, en adelante Epp. Mixt., II, p. 472). El padre Araoz escribía al fundador que "cada noche nos congregamos [los padres Oviedo,..., Miró, rector del colegio de Valencia, y Rojas, superior de la comunidad de Zaragoza, y el propio Araoz prepósito provincial de España] para tratar de las cosas que tocan la universidad y al regimiento del colegio" (carta de Araoz a Oviedo, 7-III-1549, cfr. MHSI Epp. Mixt., II, p. 113). Añade Lucáks: "No hay duda de que las constituciones escritas por Nadal [para el colegio] de Mesina, en Roma eran consideradas mejores que las de Gandía y más útiles para el uso común de la Compañía", y cuando las envía a Palermo para la fundación de un colegio allí, dice "en este orden que nos escribe Maestro Nadal" (cfr. MHSI, Lainii Monumenta, I, p. 141). Era alto el concepto que san Ignacio tenía de Jeroni Nadal, sobre el que escribía a Jeroni Doménech el 18 de marzo de 1548: "Primeramente el M[aestro] Nadal, que va por lector de teología scholastica, es docto en ella, y en la Scrittura, y en la positiva [theología]: tiene cognitión de decretos y concilios, etc. El mesmo es docto en mathemáticas, que las ha leydo en Paris (...) y seydo diligente en ellas. Es asímesmo docto en artes, y en letras de humanidad, latinas, griegas y hebreas, como allá verá por esperientia" (MHSI, Monumenta Ignatiana Epistolae, desde ahora MI, Epp., II, pp. 2526, citado en MHSI 92, Mon. Paed., I, p. 17).

Nadal en cinco años escribió tres documentos esenciales para conocer el primer desarrollo de la educación jesuita: Constitutiones Collegii Messanensis, también conocida como Ratio studiorum Collegii Messanae (1548), De studiis generalis dispositione et ordine (1552), habiendo dejado ser rector de la universidad de Mesina ese año y estando ya en Roma para apoyar el comienzo del colegio de esa ciudad fundado el año anterior, y Regulae de scholis collegiorum (1553), cuando había de viajar a España y Portugal para presentar y declarar las Constituciones de la Compañía. En ese viaje visitó todos los colegios portugueses y españoles, a los que adaptó las Reglas que llevaba ya traducidas al castellano ("regulas romanas illis colegiis accomodavit", se lee en un texto del propio Nadal y en el capítulo 3 del año 1553 del Chronicon de Juan de Polanco).
En los tres documentos Nadal subrayó la formación humanística a partir de un más que notable conocimiento de la lengua latina que se ordena en ciclos y niveles: "omnes loquantur latine utcunque, et sit qui notet non loquentes latine (todos hablen en latín sea como sea, y haya alguien que anote quiénes no hablan en latín)" (MHSI 92, p. 22). La formación religiosa es propuesta como primera base del resto de la formación que ha de recibirse: "ut in studiis primum locum pietas obtineat, (...) si primo ratio pietatis christianae et morum honestorum constituatur (para que la piedad sea lo primero en los estudios, [de forma acomodada a los estudiantes, principalmente entonces los estudios de los preceptores, y después los de los estudiantes han de ser ordenados], en primer lugar se establece el orden de la piedad cristiana y de las costumbres honestas)" (MHSI 92, p. 136). Desde el principio, pues, Nadal subrayaba la integralidad de una formación humanística y al mismo tiempo (simul) religiosa, de la piedad y las letras.

\section{EL MODELO DE COLEGIO Y UNIVERSIDAD JESUITA}

En la Ratio studiorum que Diego de Ledesma escribió para el colegio de Roma se lee:

\footnotetext{
"Scholae literariae hominum generi, in republica vero Christiana et Christi Ecclesia, maxime sunt necessariae, tum [1]ad multas vitae huius commoditates, tum [2]ad rectam rerum publicarum gubernationem et leges, tum [3]ad naturae ipsius rationalis ornatum et splendorem ac perfectionem, tum [4] demum, quod maius est, ad Dei fidem et religionem docendam, defendendam et propagandam; et ut homines ad suum tandem finem ultimum commodius et facilius perducantur; de quibus primo dicendum, quae et quaes sint, en in quo differant" (MHSI 107, pp. 528ss), titulada Ratio studiorum Collegii Romani (Liber primus: De linguarum studio, ratione et ordine) $)^{1}$.
}

A partir de este texto se ha formulado el conocido como Paradigma Ledesma-Kolvenbach, que yo me inclinaría por llamar Paradigma Ledesma-KolvenbachAgúndez pues ha sido Melecio Agúndez, jesuita, entonces coordinador de formación universitaria en UNIJES (universidades y centros superiores de los jesuitas en España) y editor de los discursos universitarios del superior general de los jesuitas (Kolvenbach, 2008), quien en 2008 logró esta síntesis.

En la pedagogía ignaciana convergen tradiciones diferentes a las que antes me he referido, como quedó bien establecido por la tesis doctoral del historiador jesuita catalán Gabriel Codina i Mir: escolástica en 
plena ebullición renacentista, humanismo literario, prácticas e intuiciones de clérigos reformadores así como de colegios luteranos o calvinistas, humanismo italiano y humanismo flamenco a través de los hermanos de la vida común y de la devotio moderna que, esta última, no olvidemos, tanto había influido en la espiritualidad ignaciana ya desde los comienzos de Montserrat y Manresa (Cataluña), por la difusión de aquella corriente realizada por el abad reformador García de Cisneros, como ha estudiado con profundidad el jesuita catalán Javier Melloni.

La síntesis que se expresa por el lema utilitas-humanitas-iustitia-fides (cfr. Agúndez Agúndez, 2008, pp. 589762) que recoge todas las intuiciones del citado texto de Diego Ledesma, podríamos desplegarla en algunas de las siguientes características del modelo jesuita:

a) Globalidad. Ignacio propuso un sistema pedagógico con un itinerario que avanzaba desde la enseñanza de la gramática, el nivel más elemental en aquel tiempo, hasta el doctorado, un sistema global, caracterizado por su flexibilidad. Esta primera innovación en relación con el sistema medieval era esencial. Éste se concebía desde el olvido de la primera etapa y la rigidez de la segunda, y centraba todo su interés en la tercera, la enseñanza superior. Los primeros jesuitas pensaban sus colegios y universidades como un lugar único en que los adolescentes de doce años (y en algunos lugares más jóvenes) empezaban con el aprendizaje de la lengua, y los jóvenes estudiantes avanzaban sin solución de continuidad hacia la formación universitaria (Artes) y las posteriores especializaciones (Teología, uno de los dos Derechos o Medicina). Este modelo sirvió después a algunas escuelas parroquiales y a otras muchas órdenes y congregaciones docentes.

b) Progresividad. A partir de la experiencia humanista flamenca (devotio moderna y otras), el colegio jesuita se ordenará progresivamente en un sistema de clases y de división del trabajo intelectual. El colegio se convierte así en un medio que responde perfectamente al nuevo modelo cultural burgués de orden y de eficiencia, tanto en los aspectos culturales y sociales como en los técnicos. Con el colegio y la periodización en etapas temporales que implica, sin temor a exagerar, podríamos decir que apareció una nueva etapa entre la infancia y la edad del trabajo: la juventud escolarizada, la adolescencia. Los jóvenes fueron organizados en clases o cursos, con un programa adaptado a cada etapa. c) Integralidad. Es conveniente poner en relación muy directa el nuevo enfoque de la espiritualidad engendrado por la devotio, al que ya nos hemos referido, y el humanismo integral que da solidez a la concepción del colegio jesuita. En este punto el influjo de la devotio resulta decisivo. Para ésta, la enseñanza no puede ser sólo intelectual, sino que debe integrar los aspectos de la piedad cristiana (pietas ac litterae, que condujera a la formación de "hombres sabios y piadosos", como escribió san Ignacio en 1551 al duque de Baviera). Ésta es una diferencia esencial entre el humanismo renacentista y el humanismo devoto que influyó a Ignacio en los colegios de la universidad de París, aunque sin llegar a los extremos rigoristas y ascéticos propios de los hermanos de la vida común, que también había conocido en el colegio de Montaigu de París. Esta integralidad pretendida se complementa con la combinación de enseñanza oral y escrita, de enseñanza colectiva y personal. El trabajo a partir de las fuentes, de los textos, el trabajo en la habitación, dos características de la enseñanza renacentista fueron incorporadas al modelo integral del colegio jesuita. Los "ejercicios", síntesis de ese carácter personal y escrito, serán una característica de ese modelo, que también combinará los sistemas de emulación y de ayuda mutua entre los alumnos.

d) Secularidad. El modelo de colegio jesuita se adaptó con fuerza a una sociedad con graves divisiones religiosas. El modelo a seguir ya no se centra en la formación de los clérigos, sino en la de todos los cristianos, concepción muy enraizada en la devotio moderna. En una Europa destrozada religiosamente la clave de los colegios residirá en la formación de seglares instruidos y devotos, junto a la contribución directa a la formación y reforma del clero allá donde sea posible, y a la configuración de un colegio como lugar él mismo de resistencia frente a la ruptura religiosa (el mapa de los colegios en la Europa central reformada), de reforma interior y de diálogo cultural. Este traumatismo europeo llevó a los seglares a distanciarse del clero decadente ("es Pablo III quien recibe en audiencia al grupo de jesuitas destinados al colegio de Mesina en 1548" -escribía con humour Kolvenbach en 2001- dado que en Sicilia (de creer a un narrador) "iexiste en las filas del clero tal ignorancia que, de no verlo, no podría imaginarse!"), lo que conllevaba la exigencia de adquisición de cultura. 
e) Unidad. La articulación de los saberes y los contenidos de la enseñanza de las letras humanas recibió con el colegio jesuita un nuevo estatuto más preciso: los autores clásicos debían estar en la base del aprendizaje de la lengua, la escritura y la reflexión, según las ideas pedagógicas de Erasmo de Rotterdam y del judío valenciano Luis Vives. Como es bien sabido, y hemos considerado en el capítulo precedente al tratar de los diferentes documentos fundacionales incluida la Ratio de 1599 , el estudio de los clásicos fue constante desde el comienzo en los colegios de la Compañía, aunque con modulaciones diferentes, como podemos comparar al analizar las dos Ratio, la de 1586, la aún renacentista, y la más barroca y definitiva de 1599, la de Acquaviva.

f) Gratuidad. Ante las nuevas necesidades de nobles y burgueses de los medios urbanos, las demandas de fundar un sistema educativo que atienda la necesidad de formación de las nuevas élites, los primeros jesuitas propusieron un sistema exigente, caracterizado por la excelencia. Sin embargo la enseñanza durante los dos largos siglos de la primera Compañía fue una enseñanza gratuita, concebida como instrumento de promoción social, pero no elitista. Un colegio jesuita respondía a las aspiraciones de las nuevas capas sociales dinámicas, a las que ofrecía un saber al servicio de una sociedad en transformación continua. Un colegio jesuita era un lugar de exigencia, de excelencia.

Estas notas características de la educación jesuita llevaron a un modelo de colegio y de universidad que podríamos reconocer o presentar en torno a estos tres verbos: enseñar, saber, creer. Éste es el trilema que emplaza a las universidades jesuitas como respuesta realizada desde una nueva matriz epistemológica (Senent de Frutos, 2014) que elabora desde una acomodación discernida un modelo de acción apostólica y social de reforma hacia fuera, de reforma móvil no tanto en el centro a conservar y estructurar (a "ordenar y disciplinar"), como en la periferia a descubrir y configurar. El colegio y la universidad jesuita no son meros lugares de enseñanza/aprendizaje, sino lugares de producción y de difusión de un modelo integral (integrado e integrador) de enseñar, saber, creer, por medio de un sistema metódico, la ratio.

Hemos llamado a éste modelo, un modelo pedagógico integral y universal, porque como ha quedado apuntado, integra todos las dimensiones socio-antropológicas, en un único modelo que es único y plural, articulado y flexible. Esto supone una antropología integral en la base de esta concepción educativa, que es la que se recoge en la ratio, como hemos indicado en páginas anteriores. El centro educativo ignaciano -colegio o universidad- es, como hemos sugerido un lugar de educación: enseñanza / aprendizaje, un lugar de producción del saber y del creer, un lugar de circulación del saber y del creer y, finalmente, un lugar de animación del saber y del creer.

El éxito sorprendente de los colegios jesuitas en el siglo XVI consistió en parte en la capacidad de crear una gran red de colegios en gran parte de Europa que en su conjunto entró en el debate contemporáneo sobre el saber. Los colegios fueron el lugar de engendramiento de un saber nuevo, y no sólo un lugar de transmisión de éste. Una historia intelectual de los jesuitas durante el Renacimiento debe dar cuenta de cómo y porqué contribuyeron a engendrar la modernidad, afirma la historiadora francesa Giard. Estos colegios, como afirmaba George G. Ganss ya en 1956, tomaron una distancia crítica en relación a la vieja universidad medieval, y por ello fueron mucho más capaces de innovar, también en su distribución geográfica, que atendía los nuevos centros urbanos y ponía en vigor nuevas configuraciones del saber. No les faltaron apoyos: las peticiones de las ciudades para abrir un colegio, las dotaciones de fundaciones, las demandas de los obispos para la formación de clérigos y fieles, los príncipes que escogían a algún jesuita entre sus consejeros o como confesor o preceptor de sus hijos, reyes que les encargaban la misión de evangelizar o de encuadrar a los nuevos súbditos de ultramar, etc. Las críticas a los jesuitas, bien conocidas, existieron junto a los reconocimientos, incluso del lado reformado, pues está bien establecido el conjunto de reconocimientos y alabanzas que los cursos de Coimbra o del Colegio Romano recibieron en el siglo XVI de parte de los luteranos. En menos de un siglo la Compañía llegó a implantar una fecunda red de establecimientos de enseñanza, colegios y universidades. Junto a ellos, dentro de los mismos o en su entorno, la Compañía creó observatorios astronómicos, gabinetes científicos, bibliotecas, imprentas y editoriales, centros de difusión de los informes de las exploraciones, así como de la literatura ejemplar de los misioneros; por otra parte los colegios serán también lugar de producción de los textos de devoción y de los manuales colegiales. Alguno, como W. Ashworth, se atreve a afirmar que la Compañía, fue "la primera verdadera sociedad científica" de Europa, por delante de la Academia del Cimento o de la Royal Society (Giard, 1995, p. xxv). Como escribe sintética y sugerentemente L. Giard: 
Personas entusiastas para discutir con la Europa sabia, miembros activos de la República de las letras, de las artes y de las ciencias, espíritus abiertos para captar las consecuencias filosóficas y teológicas de las nuevas teorías, apóstoles de un saber consagrados a saber más para comprender mejor, actuar al servicio de la gloria del Dios de su fe, los jesuitas inventaron colectivamente en el Renacimiento un nuevo tipo de vida consagrada, una vida de religiosos activos, móviles, flexibles, prestos a desplazarse a otros lugares, a cambiar de tarea, a aceptar otras representaciones teóricas.

\section{CONCLUSIÓN}

En los primeros documentos de las universidades jesuitas de mediados del siglo XVI (sobre todo los de Jeroni Nadal tanto en Mesina como en Roma, como en el de Diego Ledesma en Roma) se plasmó el sistema educativo jesuita que luego conocimos como Ratio studiorum. El núcleo original de la "invención" renacentista de los primeros colegios y universidades jesuitas Ignacio no sólo dio importancia a documentos que finalmente quedarían reflejados en las Ratio de 1586 y 1599, sino que su estrategia pasó por la sustitución de Mesina por el Colegio romano como modelo (prototypum) del sistema educativo jesuita. Fue allí donde un colegio y universidad jesuita, el Colegio romano, futura Universidad gregoriana, acabó de configurarse como lugar de producción y de difusión el saber, y no solo como centro de transmisión (educativa). La modernidad expansiva del siglo XVI alcanzó un logro destacable gracias a los centros educativos jesuitas.

\section{NOTAS}

1 Con estas palabras prácticamente comienza la Ratio de Ledesma, cfr MHSI 107 (1974) 519-521. Los números de párrafos entre corchetes son del autor.

\section{BIBLIOGRAFÍA}

Agúndez Agúndez, M. (2008) (coord.). El binomio identidad y misión ante los desafíos universitarios. Revista de Fomento Social, 252.

Batllori, M. (1997). Sant Ignasi, entre l'edat mitjana i el renaixement. En Cultura $i$ finances a l'Edat Moderna (vol. VIII). Valencia: Tres i Quatre, pp. 63-82.

Giard, L. (1995). Le devoir d'intelligence, ou l'insertion des jésuites dans le monde du savoir. En L. Giard (dir.). Les jésuites à la Renaissance. Système éducatif et production du savoir. París: PUF, pp. ix-Ixxix.

Kolvenbach, P. H. (2008). Discursos universitarios. Madrid: Unijes.

Lukács, L. (ed.) (1965). Monumenta paedagogica Societatis lesu. I (1540-1556): Monumenta historica Societatis lesu, vol. 92, Roma: Institutm Historicum Societatis lesu.

Lukács, L. (ed.) (1974). Monumenta paedagogica Societatis lesu. II (1557-1572): Monumenta historica Societatis lesu [MHSI], vol. 107, Roma: Institutm Historicum Societatis lesu.
Margenat, J. M. (2010). Competentes, conscientes, compasivos y comprometidos. La educación de los jesuitas. Madrid: PPC.

Margenat, J. M. (2013). "Amen todos la pobreza como madre». Aquella tierra fértil de hombres fuertes. Manresa, 85, 335, pp. 149-160.

Maritain, J. (1936/2000). Humanisme intégral. Problémes spirituels et temporels d'une nouvelle chrétienté. París: Aubier.

Maritain, J. (1947/1988). Pour une philosophie de l'éducation. En Oeuvres complètes (vol. VII). Friburgo de Suiza - París: Universitaires - Saint-Paul, pp. 769-988.

Mongini, G. (2012). Le teologie gesuitiche delle origini. Lo spiritualismo radicale come matrice commune del dissenso e della fedeltà all'ortodossia. En Alfieri, F. y Ferlan, C. (coords.). Avventure dell'obbedienza nella Compagnia di Gesù. Teorie e prassi fra XVI e XIX secolo. Bolonia: II Mulino, pp. 19-47.
O'Malley, J. W. (1995). Los primeros jesuitas. Bilbao-Santander: Mensajero-Sal Terrae.

O'Malley, J. W. (1996). Ministerios de la primera Compañía. ¿Disciplina social o discernimiento y acomodación? CIS. Revista de espiritualidad ignaciana, 27, 81, pp. 19-35.

O'Malley, J. W. (2014). Historia de los jesuitas. Desde Ignacio hasta el presente. Bilbao: Mensajero.

Sauvé, J., Codina, G. y Escalera, J. (2001). Educación. En O’Neill, C. Diccionario Histórico de la Compañía de Jesús (vol. 2). Roma-Madrid: Institutum Historicum Societatis lesu - Universidad Pontificia Comillas, pp. 1202-1204.

Senent de Frutos, J. A. (2014). Hacia una relectura de la matriz cultural ignaciana desde nuestras necesidades civilizatorias actuales. Concordia. Internationale Zeitschrift für Philosophie, 65, pp. 25-49. 